\title{
Cochlear Implant Benefits in Deafness Rehabilitation: PET Study of Temporal Voice Activations
}

\author{
Arnaud Coez ${ }^{1-4}$, Monica Zilbovicius ${ }^{1,2}$, Evelyne Ferrary ${ }^{5-7}$, Didier Bouccara ${ }^{5-7}$, Isabelle Mosnier ${ }^{5-7}$, \\ Emmanuèle Ambert-Dahan ${ }^{6}$, Eric Bizaguet ${ }^{3}$, André Syrota ${ }^{2}$, Yves Samson ${ }^{2,8,9}$, and Olivier Sterkers ${ }^{5-7,10}$ \\ ${ }^{1}$ CEA-INSERM U797 Research Unit Neuroimaging and Psychiatry, Service Hospitalier Frédéric-Joliot, IFR49, Orsay, France; ${ }^{2}$ CEA, \\ DRM, DSV, Service Hospitalier Frédéric-Joliot, Orsay, France; ${ }^{3}$ Laboratoire de correction Auditive, Eric Bizaguet, Paris, France; \\ ${ }^{4}$ Université Paris-Sud 11, Paris, France; ${ }^{5}$ Inserm, Unit-M 867, Paris, France; ${ }^{6}$ AP-HP, Hôpital Beaujon, Service d'ORL et de Chirurgie \\ cervico-faciale, Clichy, France; ${ }^{7}$ Institut Fédératif de Recherche Claude Bernard Physiologie et Pathologie, IFR02, Paris, France; \\ ${ }^{8}$ AP-HP, Hôpital Pitié-Salpétrière, Service Urgences cérébro-vasculaires, Paris, France; ${ }^{9}$ Université Pierre et Marie Curie Paris 6 , \\ Paris, France; and ${ }^{10}$ Université Denis Diderot-Paris 7, Paris, France
}

Cochlear implants may improve the medical and social prognosis of profound deafness. Nevertheless, some patients have experienced poor results without any clear explanations. One correlate may be an alteration in cortical voice processing. To test this hypothesis, we studied the activation of human temporal voice areas (TVA) using a well-standardized PET paradigm adapted from previous functional MRI (fMRI) studies. Methods: A PET $\mathrm{H}_{2}{ }^{15} \mathrm{O}$ activation study was performed on 3 groups of adult volunteers: normal-hearing control subjects $(n=6)$ and cochlearimplanted postlingually deaf patients with $>2$ y of cochlear implant experience, with intelligibility scores in the "Lafon monosyllabic task" $>80 \%$ (GOOD group; $n=6$ ) or $<20 \%$ (POOR group; $n=6)$. Relative cerebral blood flow was measured in 3 conditions: rest, passive listening to human voice, and nonvoice stimuli. Results: Compared with silence, the activations induced by nonvoice stimuli were bilaterally located in the superior temporal regions in all groups. However these activations were significantly and similarly reduced in both cochlear implant groups, whereas control subjects showed supplementary activations. Compared with nonvoice, the voice stimuli induced bilateral activation of the TVA along the superior temporal sulcus (STS) in both the control and the GOOD groups. In contrast, these activations were not detected in the POOR group, which showed only left unilateral middle STS activation. Conclusion: These results suggest that PET is an adequate method to explore cochlear implant benefits and that this benefit could be linked to the activation of the TVA.

Key Words: auditory cortex; hearing neuroimaging; hearing loss; temporal voice area

J Nucl Med 2008; 49:60-67

DOI: 10.2967/jnumed.107.044545

Received Jun. 24, 2007; revision accepted Sep. 25, 2007.

For correspondence or reprints contact: Arnaud Coez, PharmD, Laboratoire de correction Auditive, Eric Bizaguet, 20, rue Thérèse, 75001 Paris, France.

E-mail: acoez@noos.fr

COPYRIGHT @ 2008 by the Society of Nuclear Medicine, Inc.
C ochlear implants restore an auditory sensation that can be used and integrated by the neural system of humans through the electrical stimulation of auditory nerve fibers $(1,2)$. Cochlear implantation is usually required when traditional hearing aids, which use residual ear function, fail. Most patients are able to use this new artificial code, and the social and the medical prognosis of profound deafness is now notably improved. Nevertheless, cochlear implantation performance varies widely from simple noise detection to full comprehension of speech (3-5). Many factors-including duration of deafness, age at implantation, mode of communication, duration of device use, and coding strategy - are suggested to interact with cortical map organization and to influence the final results (6-10). As a result, clinical practice recommends detection and rehabilitation of the auditory impairment as early as possible to keep the functions of brain areas specialized for auditory networks $(11,12)$.

The effectiveness of the cochlear implant is typically evaluated by a subjective score of speech intelligibility that requires previous knowledge of oral language from the tested subject as well as preserved intentional abilities. The speech intelligibility score is an integrated score, and it cannot be used to localize the site of brain dysfunction, in the case of poor results, from the peripheral implant to the integrative area of speech production. Neuroimaging techniques constitute an opportunity to describe the cortical networks engaged in cochlear implant users (13-19), to evaluate the neural consequences of electrical stimulation by the cochlear implant $(20)$, and to predict outcome $(21,22)$. These studies suggest that it may be realistic to develop a prognostic tool to gauge cochlear implant effectiveness in deaf patients.

A functional MR imagery (fMRI) paradigm has allowed the identification of bilateral temporal voice areas (TVA) in normal-hearing adults in the associative auditory cortex 
along the upper bank of the superior temporal sulcus (STS) by showing that these areas were selectively activated by voice stimuli when compared with carefully matched nonvoice stimuli. (23). Activation of the human TVA has been proposed to constitute a crucial step in cerebral voice processing, from which the different types of vocal information-speech, identity, affect—are then processed in partially segregated functional pathways (24). Other pathways for the processing of voice and speech are possible. Indeed, voice structural analysis could not be a central node on which subsequent speech recognition depended. Nevertheless, we have considered that if the acoustic cues of voice are not detected, then fine speech acoustic cues could not be analyzed. TVA activation can allow for the exploration of temporal regions engaged in voice processing without requiring higher-order processing of lexical and semantic information. In patients with a cochlear implant, this paradigm allows exploration of the voice transduction from the cochlear implant to the TVA.

The aim of this study was to test whether activation of the TVA is correlated with good intelligibility in patients with cochlear implants. We hypothesized that a difference in activation of the voice-selective brain area may be linked to a difference in the clinical intelligibility score. To test this hypothesis, we adapted a fMRI paradigm previously validated $(23,25)$ to PET because the magnet of the cochlear implant did not allow fMRI studies. To test human voice perception in cochlear implant patients of various ages and linguistic ability, we measured the relative cerebral blood flow (rCBF) by PET in 3 groups of subjects: normal-hearing persons (intelligibility score $=100 \%$ ) and implanted deaf subjects with high intelligibility scores $(>80 \%)$ or with very poor scores $(<20 \%)$. We predicted that patients with high intelligibility scores would have stronger TVA activation than patients with low intelligibility scores.

\section{MATERIALS AND METHODS}

\section{Subjects}

Eighteen right-handed adults were included in this study; they gave informed, written consent, and the Xavier-Bichat Hospital ethics committee approved the protocol. They were divided into 3 groups of 6 persons each (Table 1).

A control group consisted of normal-hearing, male volunteers, without any otologic, neurologic, or psychiatric disorders.

Twelve implanted patients who had a bilateral postlingual hearing loss were chosen considering their intelligibility score to the "Lafon monosyllabic task" at $65 \mathrm{~dB}: 1$ group ( 2 males) with a high intelligibility score $(>80 \%$, GOOD group) and 1 group (4 males) with a low intelligibility score $(<20 \%$, POOR group). Both groups had similar free-field warble tone audiometric thresholds indicating similar audibility of sounds (Table 1). All patients had been implanted (cochlear) between 1999 and 2003; 9 patients (5 in GOOD group and 4 in POOR group) had a Sprint (cochlear) processor; the others had an Esprit $3 \mathrm{G}$ (cochlear) processor. Four and 3 patients were left side implanted in the GOOD and POOR groups, respectively. The cochlear implant was used $>8 \mathrm{~h} / \mathrm{d}$ in all cases. The follow-up was $>2 \mathrm{y}$. In the GOOD group, the etiology of the hearing loss was sudden deafness $(n=2)$, progressive sensorineural hearing loss $(n=3)$, and otosclerosis $(n=1)$; the duration of sound privation was from 0 to $7 \mathrm{y}$. In the POOR group, the etiology of the hearing loss was sudden deafness $(n=2)$, bacterial meningitis $(n=3)$, and viral deafness $(n=1)$; the duration of sound privation was from to 0 to $31 \mathrm{y}$.

\section{Task and Stimuli}

Twelve PET acquisitions during passive listening were obtained: Four measurements occurred during the rest condition, 4 occurred during passive listening to voice condition, and 4 occurred during passive listening of nonvoice condition. The listening of blocs had been randomized. The voice stimuli were those used in the original fMRI study (23) and were adapted to a PET paradigm. The proportion of voice stimuli heard in the fMRI paradigm was either speech (33\%: words, non words, foreign language) or nonspeech (67\%: laughs, sighs, various onomatopoeia). The PET adaptation modified these initial proportions (30\% speech; $70 \%$ nonspeech). Initial nonvoice stimuli consisted of sounds from nature (14\%: e.g., wind, streams), animals (29\%: cries, gallops), modern human environment (37\%: cars, telephones, airplanes), and musical instruments (20\%: bells, harp, instrumental orchestra) that had also been modified by the PET paradigm (nature: $19 \%$; animals: $24 \%$; modern human environment: $47 \%$; musical instruments: $10 \%$ ). Stimuli were delivered binaurally, at a mean 65-dB SPL, by KOSS electrostatic headphones to normal-hearing patients, and monaurally, directly through the implant accessory input, at the most comfortable level to implanted patients.

TABLE 1

Clinical Data

\begin{tabular}{|c|c|c|c|c|c|c|c|c|c|}
\hline \multirow[b]{2}{*}{ Group } & \multicolumn{4}{|c|}{ Threshold (dB) } & \multirow[b]{2}{*}{ IS } & \multirow{2}{*}{$\begin{array}{l}\text { Deafness } \\
\text { duration }(\mathrm{y})\end{array}$} & \multirow{2}{*}{$\begin{array}{c}\text { Auditory } \\
\text { deprivation (y) }\end{array}$} & \multirow{2}{*}{$\begin{array}{c}\text { Cochlear implant } \\
\text { experience (y) }\end{array}$} & \multirow{2}{*}{$\begin{array}{l}\text { Left } \\
\text { implant }(n)\end{array}$} \\
\hline & $0.5 \mathrm{kHz}$ & $1 \mathrm{kHz}$ & $2 \mathrm{kHz}$ & $4 \mathrm{kHz}$ & & & & & \\
\hline GOOD IS > $>80 \%$ & $36 \pm 17$ & $34 \pm 11$ & $29 \pm 8$ & $34 \pm 11$ & $85 \% \pm 8 \% 55 \pm 11$ & $20 \pm 8$ & $3.2 \pm 3.0$ & $3.0 \pm 1.6$ & 4 \\
\hline POOR IS $<20 \%$ & $41 \pm 11$ & $37 \pm 7$ & $32 \pm 3$ & $35 \pm 7$ & $5 \% \pm 8 \% 49 \pm 15$ & $23 \pm 13$ & $10.7 \pm 15.0$ & $4.1 \pm 2.1$ & 3 \\
\hline \multicolumn{10}{|c|}{$\begin{array}{l}\text { IS }=\text { intelligibility score. } \\
\text { Implanted patients were selected and divided into } 2 \text { groups (GOOD and POOR) according to their IS (Lafon monosyllabic test). Audibility } \\
\text { f sounds, appreciated by the tonal audiometry threshold (dB), was similar in the } 2 \text { groups of implanted patients. All were right-handed and } \\
\text { lost of them had a left-sided cochlear implant. Groups of patients were matched in age and in auditory experience. Values are means } \pm \\
\text { D; } n=6 \text { in each group. }\end{array}$} \\
\hline
\end{tabular}




\section{PET Acquisition}

The $\mathrm{rCBF}$ was determined from the distribution of radioactivity measured with PET (ECAT-EXACT-HR+; Siemens AG) after bolus intravenous $\mathrm{H}_{2}{ }^{15} \mathrm{O}$ injections (26). Listeners received 12 $\mathrm{H}_{2}{ }^{15} \mathrm{O}$ injections (333 $\mathrm{MBq}$ per injection) corresponding to 12 rCBF measurements, performed at 10-min intervals. Attenuationcorrected data were reconstructed into sixty-three 2.25-mm-thick axial slices, with a resulting resolution of $4.5-\mathrm{mm}$ full width at half-maximum after reconstruction (27).

\section{Data Analysis}

The rCBF images were analyzed with statistical parametric mapping software (SPM99; Wellcome Neurological Laboratory, U.K.) used for image realignment, transformation into standard stereotactic anatomic space (28), smoothing (12 mm), and statistical analyses (29). State-dependent differences in global flow were covaried using proportional scaling. Comparisons across conditions were made with the $t$ statistic subsequently transformed into the normally distributed $z$ statistic by using a multistudy design. The resulting $z$ maps were thresholded at $P<0.001$. Three statistical analyses of activation were performed: A within-group comparison of activation for listening to voice or nonvoice sounds versus the rest condition, a within-group comparison between the voice condition versus the nonvoice condition, and a betweengroup comparison of these comparisons across conditions.

\section{RESULTS}

\section{Auditory Activation: Nonvoice Sounds Versus Silence}

When compared with silence, the nonvoice stimuli elicited bilateral activation in the temporal regions. In the 3 groups (Table 2; Fig. 1A), the stimuli were located from the anterior to the posterior part of STS in the bank of the superior temporal gyrus (STG) and the middle temporal gyrus (MTG) (Brodmann areas: BA21, BA22, BA42).

Comparison of the auditory activation maps between the 3 groups showed supplementary bilateral activation in control patients compared with implanted patients $(P<$ 0.001; Table 3; Fig. 2A). The main plots were located bilaterally in the posterior part of the STS. No difference was observed between the GOOD and POOR groups of implanted patients.

TABLE 2

Coordinates, Size, and Z Score of Areas Activated by Nonvoice Compared with Silence in Each of 3 Groups: Normal Hearing, GOOD Cochlear Implanted, and POOR Cochlear Implanted

\begin{tabular}{|c|c|c|c|c|c|c|c|c|c|}
\hline \multirow[b]{2}{*}{ Area } & \multicolumn{4}{|c|}{ Normal hearing } & \multicolumn{4}{|c|}{ Voxel } & \multirow{2}{*}{$\frac{\text { Cluster }}{P \text { corrected }}$} \\
\hline & $x$ & $y$ & $z$ & Size & $Z$ & $t$ & $\%$ & $P$ corrected & \\
\hline STS, anterior (BA21) & 54 & -10 & -4 & & $>10$ & 12.14 & 8.8 & $<0.0001$ & $<0.0001$ \\
\hline STS posterior (BA42/BA22) & 58 & -24 & 8 & 5,269 & $>10$ & 13.9 & 10.2 & $<0.0001$ & \\
\hline Right uncus (BA28) & 30 & 2 & -18 & 104 & 4.29 & 4.40 & 2.9 & 0.113 & NS \\
\hline STS, anterior (BA21) & -50 & 4 & -10 & 209 & 7.54 & 8.16 & 5.8 & $<0.0001$ & $<0.0001$ \\
\hline STS, posterior (BA22) & -64 & -34 & 12 & & $>10$ & 9.24 & 6.0 & $<0.0001$ & \\
\hline \multirow[t]{2}{*}{ STS, posterior (BA22) } & -42 & -26 & 6 & 4,375 & $>10$ & 9.99 & 7.7 & $<0.0001$ & \\
\hline & \multicolumn{4}{|c|}{ Implanted GOOD } & \multicolumn{4}{|c|}{ Voxel } & Cluster \\
\hline Area & $x$ & $y$ & $z$ & Size & $Z$ & $t$ & $\%$ & $P$ corrected & $\overline{P \text { corrected }}$ \\
\hline STS, middle (BA21) & 60 & 0 & -6 & & 5.91 & 6.21 & 4.7 & $<0.0001$ & $<0.0001$ \\
\hline STS posterior (BA42/BA22) & 64 & -30 & 4 & 3,570 & $>10$ & 9.60 & 7.4 & $<0.0001$ & \\
\hline Right distal frontal gyrus (BA6) & 8 & 12 & 54 & 60 & 3.67 & 3.74 & 2.6 & 0.655 & NS \\
\hline STS, anterior (BA21) & -48 & 0 & -8 & & 4.89 & 5.06 & 3.8 & 0.010 & 0.006 \\
\hline STS, middle (BA21) & -64 & -14 & 0 & & 5.22 & 5.42 & 3.5 & 0.002 & $<0.0001$ \\
\hline \multirow[t]{2}{*}{ STS, posterior (BA22) } & -56 & -26 & 4 & 2,943 & 6.82 & 7.27 & 5.7 & $<0.0001$ & \\
\hline & \multicolumn{4}{|c|}{ Implanted POOR } & \multicolumn{4}{|c|}{ Voxel } & Cluster \\
\hline Area & $x$ & $y$ & $z$ & Size & $Z$ & $t$ & $\%$ & $P$ corrected & $\overline{P \text { corrected }}$ \\
\hline STS, anterior (BA21) & 58 & -16 & 0 & & 5.67 & 5.99 & 4.6 & $<0.0001$ & $<0.0001$ \\
\hline STS, middle (BA21) & 58 & -2 & -6 & & 5.50 & 5.79 & 4.4 & 0.001 & \\
\hline STS posterior (BA42/BA22) & 60 & -30 & 2 & 3,396 & 6.61 & 7.03 & 5.2 & $<0.0001$ & \\
\hline STS, anterior (BA21) & -48 & -10 & -6 & & 3.49 & 3.48 & 2.8 & 0.888 & NS \\
\hline STS, posterior (BA22) & -44 & -34 & 12 & & 3.35 & 3.40 & 2.6 & 0.934 & NS \\
\hline STS, posterior (BA22) & -48 & -26 & 6 & 313 & 3.82 & 3.90 & 3.3 & 0.476 & \\
\hline
\end{tabular}

Coordinates (in standard stereotactic space (28)) of voxels corresponding to local maxima of $Z$ value, above $Z=4.75(P<0.001)$ within each focus of activation: $x=$ distance $(\mathrm{mm})$ to right $(+)$ or left $(-)$ of midsagittal line; $y=$ distance anterior $(+)$ or posterior $(-)$ to vertical plane through anterior commissure; $z=$ distance above $(+)$ or below $(-)$ intercommissural (AC-PC) line; STS = superior temporal sulcus; $\mathrm{NS}=$ not significant; $\%=$ relative $\mathrm{rCBF}$ change $(\%)$.

Approximate Brodmann numbers (BA) associated with anatomic regions are given in parentheses. Size refers to number of voxels in a given cluster (voxel size in mm, $2 \times 2 \times 2$ ), for statistical parametric mapping SPM $(Z)$ map thresholds at $\mathrm{T}=3.13(P<0.001$, uncorrected) and then corrected for multiple comparisons $(P<0.05)$. 
A

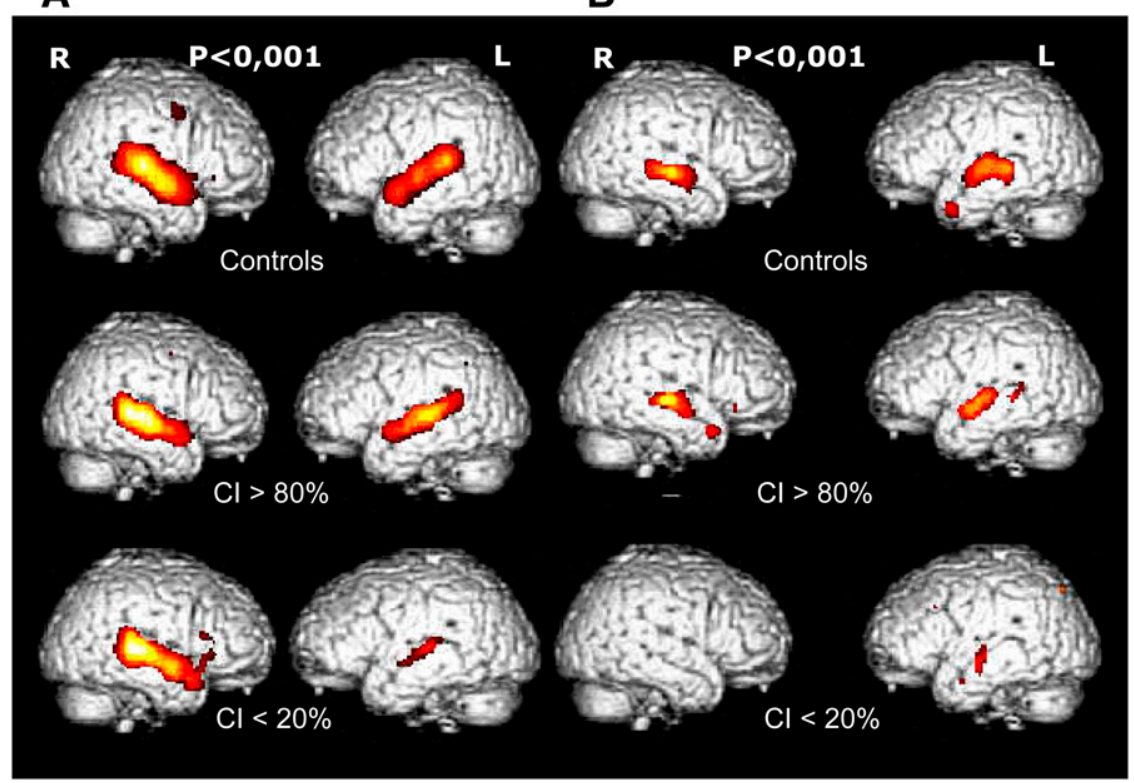

FIGURE 1. Intragroup contrasts. Location of activation peaks when listening to nonvoice compared with silence $(A)$ and voice compared with nonvoice sound $(B)$ in 3 groups (normal hearing and cochlear implanted patients) at $P<0.001$ are shown in a lateral view of both hemispheres.

\section{Voice-Selective Activations: Voice Versus Nonvoice Sounds}

In the control group, the activation maps elicited by the voice versus nonvoice contrast $(P<0.001)$ were bilaterally located, along the middle $( \pm 66,-10,-6)$ and the posterior part of the STS ( $\pm 64,-30,-2$ ) (Table 4; Fig. 1B), close to the TVA identified by previous fMRI results $([ \pm 62,-14$, $0]$ and $[56,-30,6])$ (23). In the GOOD group, the activations were very similar to those of the control subjects. As shown in Table 4 and Figure 1B, the activations were also bilateral and located in the middle and the posterior part of the STS. Conversely, in the POOR group, the activations were restricted to a single left peak of activation in the middle part of the STS.
The group comparison (Table 5; Fig. 2B) confirmed that TVA activation was significantly and bilaterally reduced in the POOR group compared with that of the control subjects, whereas there were no significant differences between the control and GOOD groups. In addition, when the activations between the 2 implanted groups were compared, there was a significant right supplementary activation in the anterior STS in the GOOD group compared with that of the POOR group $(P<0.001)$.

\section{DISCUSSION}

This study shows a link between TVA activation and the speech intelligibility score in cochlear-implanted patients.

\section{A}

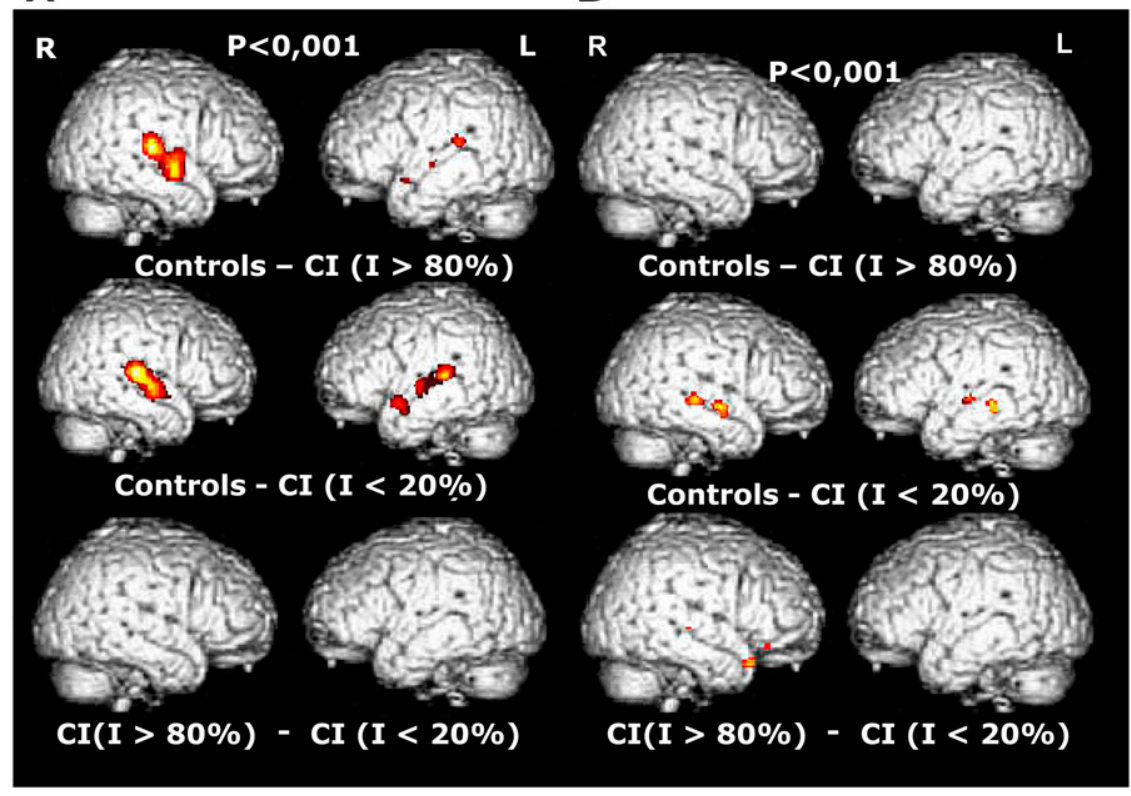

FIGURE 2. Intergroup contrasts. Location of differences of activation peaks between 3 groups (normal hearing and cochlear implanted patients) when listening to nonvoice compared with silence (A) and voice compared with nonvoice sounds $(\mathrm{B})$ at $P<0.001$ are shown in a lateral view of both hemispheres. 
TABLE 3

Localization of Significant Differences Between 3 Groups Listening to Nonvoice Compared with Silence Test

\begin{tabular}{|c|c|c|c|c|c|c|c|c|}
\hline \multirow[b]{2}{*}{ Area } & \multicolumn{4}{|c|}{ Normal hearing vs. implanted GOOD } & \multicolumn{3}{|c|}{ Voxel } & \multirow{2}{*}{$\frac{\text { Cluster }}{P \text { corrected }}$} \\
\hline & $x$ & $y$ & $z$ & Size & $Z$ & $t$ & $P$ corrected & \\
\hline STS, anterior (BA21) & 50 & -10 & -6 & & 4.45 & 4.57 & 0.069 & NS \\
\hline STS, posterior (BA42) & 54 & -24 & 10 & 1,061 & 5.04 & 5.22 & 0.005 & 0.003 \\
\hline STS, middle (BA21) & -46 & -12 & -2 & & 3.30 & 3.35 & 0.956 & NS \\
\hline STS, posterior (BA22) & -64 & -34 & 16 & 71 & 3.82 & 3.9 & 0.472 & NS \\
\hline \multirow[t]{2}{*}{ STS, posterior (BA22) } & -40 & -28 & 10 & 76 & 3.37 & 3.42 & 0.925 & NS \\
\hline & \multicolumn{4}{|c|}{ Normal hearing vs. implanted POOR } & \multicolumn{3}{|c|}{ Voxel } & Cluster \\
\hline Area & $x$ & $y$ & $z$ & Size & $Z$ & $t$ & $P$ corrected & $\overline{P \text { corrected }}$ \\
\hline STS, middle (BA21) & 50 & -12 & -4 & & 5.26 & 5.47 & 0.002 & $<0.0001$ \\
\hline STS, middle (BA42) & 70 & -16 & 6 & & 4.69 & 4.84 & 0.029 & \\
\hline STS, posterior (BA42) & 56 & -24 & 8 & 1,398 & 6.18 & 6.51 & $<0.0001$ & \\
\hline STS, anterior (BA21) & -50 & 4 & -10 & 190 & 4.01 & 4.10 & 0.288 & NS \\
\hline STS, posterior (BA22) & -64 & -34 & 12 & 959 & 4.75 & 4.90 & 0.019 & 0.019 \\
\hline STS, posterior (BA22) & -40 & -20 & 4 & & 4.72 & 4.87 & 0.021 & 0.004 \\
\hline
\end{tabular}

Coordinates (in standard stereotactic space (28)) of voxels corresponding to local maxima of $Z$ value, above $Z=4.75(P<0.001)$ within each focus of activation: $x=$ distance $(\mathrm{mm})$ to right $(+)$ or left $(-)$ of midsagittal line; $y=$ distance anterior $(+)$ or posterior $(-)$ to vertical plane through anterior commissure; $z=$ distance above $(+)$ or below $(-)$ intercommissural (AC-PC) line; STS = superior temporal sulcus; NS = not significant.

Approximate Brodmann numbers (BA) associated with anatomic regions are given in parentheses. Size refers to number of voxels in a given cluster (voxel size in mm, $2 \times 2 \times 2$ ), for statistical parametric mapping SPM $(Z)$ map thresholds at $\mathrm{T}=3.13(P<0.001$, uncorrected) and then corrected for multiple comparisons $(P<0.05)$. No different clusters were observed between GOOD and POOR groups.

Hence, in the voice versus nonvoice contrast, the activations were preserved in the GOOD group but were markedly reduced in the POOR group. Conversely, the activations induced by nonvoice stimuli were reduced at the same extent in both groups of implanted patients, consistently with their similar tonal audiometric thresholds.

\section{TVA and Brain Imaging}

A meta-analysis of neuroimaging studies with normalhearing subjects (30) suggested that acoustical parameters of speech useful to language intelligibility are specifically able to induce bilateral temporal activations in a region that has been called the "speech-sensitive auditory cortex." These activations appear to be unrelated to linguistic analysis but, rather, to be associated with the speech signal per se $(31,32)$. The TVA has been defined as the cortical areas activated by the contrast of carefully matched voice versus nonvoice stimuli (23) and belongs to this associative auditory cortical network, which is centered on the upper bank of the STS.

The activation of the TVA was observed in the control subjects of this PET study, but the pattern differed from that of the original fMRI study. In the latter (23), a larger activation of the right TVA was reported, whereas we found a slight reverse left asymmetry. This discrepancy may be explained by the modified repartition of acoustic cues of the initial fMRI paradigm in the time and in the spectral domain, which is known to induce different patterns of activation (33). Indeed, no hemisphere differences were recorded in the fMRI study when stimuli were spectral filtered (23).
Interestingly, the GOOD group had the same pattern of activation as that of the control subjects, suggesting that the cochlear implant transduction had correctly transmitted the acoustic cue proportions even if the stimulation was monaural in the implanted group and binaural in the control group. Some studies (34) found stronger activation in the contralateral size of the implant. In this study, in the GOOD group, $66 \%$ of patients were left-implanted and the main effect was also left-sided as in the control group. Moreover, the POOR group with 50\% of left-implanted patients presented only left activations. The TVA activations did not seem to depend on the size of the implantation. This paradigm did not require any language knowledge and any active participation. Rather, it avoided testing of the entire audiophonatory loop in contrast to the intelligibility task. It allowed more specific testing of the role of the cochlear implant in the transduction of sound information without testing increased activity in areas of the inferior prefrontal cortex when listening to speech and nonspeech stimuli (35). This PET paradigm, without speech stimuli, would allow the direct testing of the processing of "structural encoding" (36), specific for the auditory system, even with infants. TVA activations were preliminary to the oral intelligibility brain network and were close to the activations induced by intelligible speech in normal-hearing subjects (37).

\section{Electrical Cochlear Implant Stimulation and TVA}

In both cochlear implant groups the activations induced by nonvoice stimuli were reduced, a possible correlate of diminished audibility, which was altered to the same extent 
TABLE 4

Coordinates, Size, and Z Score of Areas Activated by Voice Compared with Nonvoice Sounds in Each of 3 Groups: Normal Hearing, GOOD Cochlear Implanted, and POOR Cochlear Implanted

\begin{tabular}{|c|c|c|c|c|c|c|c|c|c|}
\hline \multirow[b]{2}{*}{ Area } & \multicolumn{4}{|c|}{ Normal-hearing group } & \multicolumn{4}{|c|}{ Voxel } & \multirow{2}{*}{$\frac{\text { Cluster }}{P \text { corrected }}$} \\
\hline & $x$ & $y$ & $z$ & Size & $Z$ & $t$ & $\%$ & $P$ corrected & \\
\hline STS, middle (BA21) & -62 & -14 & 0 & 1,137 & 6.72 & 7.15 & 4.9 & $<0.0001$ & $<0.0001$ \\
\hline STS, posterior (BA21) & -64 & -30 & -2 & & 5 & 5.18 & 4.4 & 0.006 & \\
\hline STS, middle (BA21) & 66 & -10 & -6 & 1,028 & 5.56 & 5.80 & 4.8 & $<0.0001$ & $<0.0001$ \\
\hline \multirow[t]{2}{*}{ STS, posterior (BA21) } & 62 & -30 & 0 & & 4.56 & 4.69 & 3.5 & 0.029 & 0.029 \\
\hline & \multicolumn{4}{|c|}{ Implanted GOOD } & \multicolumn{4}{|c|}{ Voxel } & Cluster \\
\hline Area & $x$ & $y$ & $z$ & Size & $Z$ & $t$ & $\%$ & $P$ corrected & $\overline{P \text { corrected }}$ \\
\hline STS, anterior (BA21) & -60 & -6 & -8 & & 4.39 & 4.51 & 3.3 & 0.078 & NS \\
\hline STS, middle (BA21) & -66 & -16 & 2 & 501 & 4.39 & 4.51 & 2.8 & 0.078 & NS \\
\hline STS, middle (BA21) & 70 & -14 & -2 & & 4 & 4.09 & 3.4 & 0.295 & NS \\
\hline STS, posterior (BA21) & 60 & -24 & 0 & 533 & 4.2 & 4.30 & 3.3 & 0.157 & NS \\
\hline \multirow[t]{2}{*}{ STG (BA38) } & 46 & 12 & -26 & 79 & 3.75 & 3.82 & 2.9 & 0.561 & NS \\
\hline & \multicolumn{4}{|c|}{ Implanted POOR } & \multicolumn{4}{|c|}{ Voxel } & Cluster \\
\hline Area & $x$ & $y$ & $z$ & Size & $Z$ & $t$ & $\%$ & $P$ corrected & $\overline{P \text { corrected }}$ \\
\hline STS, middle (BA21) & -68 & -16 & -6 & 51 & 3.54 & 3.61 & 2.5 & 0.787 & NS \\
\hline
\end{tabular}

Coordinates (in standard stereotactic space (28)) of voxels corresponding to local maxima of $Z$ value, above $Z=4.75(P<0.001)$ within each focus of activation: $x=$ distance $(\mathrm{mm})$ to right $(+)$ or left $(-)$ of midsagittal line; $y=$ distance anterior $(+)$ or posterior $(-)$ to vertical plane through anterior commissure; $z=$ distance above $(+)$ or below $(-)$ intercommissural (AC-PC) line; STG $=$ superior temporal gyrus; STS = superior temporal sulcus; NS = not significant; \% = relative rCBF change (\%).

Approximate Brodmann numbers (BA) associated with anatomic regions are given in parentheses. Size refers to number of voxels in a given cluster (voxel size in $\mathrm{mm}, 2 \times 2 \times 2$ ), for statistical parametric mapping SPM $(Z)$ map thresholds at $\mathrm{T}=3.13(P<0.001$, uncorrected) and then corrected for multiple comparisons $(P<0.05)$.

in both groups as shown by the subjective audiometric results. Despite this reduced audibility, human voice stimuli without lexical or semantic information were able to induce TVA activation along the upper banks of both hemispheres of the STS, only when intelligibility was restored by a cochlear implant. Bilateral peaks of activations were found within $9.8 \mathrm{~mm}$ of those observed in the control subjects, and the level of activation was not significantly reduced when compared with that of the control subjects. A previous neuroimaging study (15) also presented evidence of

TABLE 5

Localization of Significant Differences Between 3 Groups Listening to Voice Compared with Nonvoice Sounds

\begin{tabular}{|c|c|c|c|c|c|c|c|c|}
\hline \multirow[b]{2}{*}{ Area } & \multicolumn{4}{|c|}{ Normal hearing vs. implanted POOR } & \multicolumn{3}{|c|}{ Voxel } & \multirow{2}{*}{$\frac{\text { Cluster }}{P \text { corrected }}$} \\
\hline & $x$ & $y$ & $z$ & Size & $Z$ & $t$ & $P$ corrected & \\
\hline MTG (BA21) & -66 & -32 & -6 & 61 & 3.75 & 3.83 & 0.552 & 0.696 \\
\hline STS posterior (BA21) & 62 & -30 & 0 & 70 & 3.58 & 3.65 & 0.747 & 0.643 \\
\hline \multirow{2}{*}{ STS anterior (BA21) } & 66 & -8 & -6 & 89 & 3.67 & 3.74 & 0.652 & 0.539 \\
\hline & \multicolumn{4}{|c|}{ Implanted GOOD vs. implanted POOR } & \multicolumn{3}{|c|}{ Voxel } & Cluster \\
\hline Area & $x$ & $y$ & $z$ & Size & $Z$ & $t$ & $P$ corrected & $\overline{P \text { corrected }}$ \\
\hline STG (BA38) & 48 & 20 & -26 & 40 & 3 & 3.68 & 0.721 & NS \\
\hline
\end{tabular}

Coordinates (in standard stereotactic space (28)) of voxels corresponding to local maxima of $Z$ value, above $Z=4.75(P<0.001)$ within each focus of activation: $x=$ distance $(\mathrm{mm})$ to right $(+)$ or left $(-)$ of midsagittal line; $y=$ distance anterior $(+)$ or posterior $(-)$ to vertical plane through anterior commissure; $z=$ distance above (+) or below (-) intercommissural (AC-PC) line; STG = superior temporal gyrus; STS = superior temporal sulcus; MTG = middle temporal gyrus; NS = not significant.

Approximate Brodmann numbers (BA) associated with anatomic regions are given in parentheses. Size refers to number of voxels in a given cluster (voxel size in mm, $2 \times 2 \times 2$ ), for statistical parametric mapping SPM $(Z)$ map thresholds at $\mathrm{T}=3.13(P<0.001$, uncorrected) and then corrected for multiple comparisons $(P<0.05)$. No different clusters were observed between the normal hearing and implanted GOOD groups. 
significant supplementary temporal activations to words compared with noise in patients with cochlear implants. Conversely, the selective TVA activation was markedly reduced in the POOR group. A single very small cluster of activation was found in the left STS, and the activation was bilaterally reduced significantly when compared with that of the control subjects. However, it should be noted that the direct comparison of the GOOD and POOR groups revealed only a single peak of significant difference in the left STG. This may be due to limited statistical power. Nevertheless, interestingly, a study (23) found a decreased response to voice when stimuli were degraded by spectral filtering in normal-hearing subjects. Therefore, activation of voice recognition networks of the STS may depend on sufficient acoustic information. It may not be available to an extent that gives rise to TVA activity in the POOR group.

\section{Poor Results Analysis}

Subjects with low comprehension perceived sounds but lacked the capacity for sufficient sound analysis to extract human voice acoustic parameters from other sounds. A cochlear implant patient would also fail to understand speech if the element of the speech signal could not be routed correctly to the region executing the fit. Because of the low activation of TVA described in this group of cochlearimplanted patients, one could assume that the poor intelligibility score of these patients might be related to the low voice recognition. This insufficient quality of soundparameter discrimination could be linked to neural dysfunction between the cochlear implant and the TVA, as suggested by the anatomopathologic studies about ganglion cell degeneration induced by the hearing loss. Spiral ganglion cell counts were highest in individuals who were deafened by aminoglycoside toxicity and by sudden idiopathic deafness and were lowest in those deafened by genetic deafness or bacterial meningitis (38). This is in line with recent clinical cochlear implant analysis (39) showing worse results for cochlear implantation after bacterial meningitis. In the present study, the main etiology in the POOR group was effectively meningitis. Another explanation must be considered. Vocal information processing might be dissociated in different functionally independent systems. Cortical regions involved in processing of different types of vocal information is likely to interact to build increasingly abstract representations (36), and TVA activation may be dependent on the recruitment of other speechprocessing systems. This is similar to the functional cortical map reorganization study of Wernicke area (15), in which the contribution of visual areas to speech comprehension (14) and an enhanced intentional resources network in rehabilitated cochlear implant patients contributed to speech processing. Our patients were all postlingually deaf with implanted cochlea of at least $2 \mathrm{y}$, and they all communicated orally without sign language. They all had strong lipreading communication skills but, in the POOR group, the oral communication in daily life depended much more strongly on this ability, especially for patients with a duration of 30-y sound privation. There were no reasons to suspect that cochlear-implanted patients had a problem in TVA activation, unless one hypothesized a reorganization of the TVA due to the longer period of deafness in this group of patients. Also, the abstract representation of voice in the TVA could depend, in the POOR group, on supplementary sensory input helpful to complete degraded voice information along the auditory neural pathways. This PET paradigm tested more directly the auditory system per se and its ability to deal with voice perception analysis without testing other complementary modalities or intentional brain networks, as suggested by the localization of activations in the TVA, even if these postlingual deaf patients had developed robust lip-reading skills to communicate orally.

\section{CONCLUSION}

A PET activation study appeared adequate to explore the benefits of cochlear implants. Cochlear implant effectiveness appeared to be linked to the patency of the neural auditory pathway and to TVA activation. A complementary PET study must be performed to establish the correlation between the intelligibility score and the degree of rCBF increase to voice versus nonvoice stimuli at an individual stage. This paradigm could then be used to establish comparisons between the therapeutic strategies of auditory rehabilitation.

\section{ACKNOWLEDGMENTS}

We thank Pascal Belin for support, advice, and useful comments on the manuscript; Cochlear Ltd. for the financial support of PET examinations; the engagement of the Beaujon Hospital clinical team, and the motivation of their patients in this study. This research was supported by the Institut National de la Santé et de la Recherche Médicale, Inserm, France, and the Commissariat à l'Energie Atomique, CEA, France. The authors declare that they have no competing financial interests.

\section{REFERENCES}

1. House WF, Urban J. Long term results of electrode implantation and electronic stimulation of the cochlea in man. Ann Otol Rhinol Laryngol. 1973;82:504-517.

2. Clark GM. Cochlear implant surgery for profound or total hearing loss. Med J Aust. 1978;2:587-588.

3. Gibson WP, Rennie M, Psarros C. Outcome after cochlear implantation and auditory verbal training in terms of speech perception, speech production and language. Adv Otorhinolaryngol. 2000;57:250-253.

4. Makhdoum MJ, Snik AF, van den Broek P. Cochlear implantation: a review of the literature and the Nijmegen results. J Laryngol Otol. 1997;111:1008-1017.

5. van Dijk JE, van Olphen AF, Langereis MC, Mens LH, Brokx JP, Smoorenburg GF. Predictors of cochlear implant performance. Audiology. 1999;38:109-116.

6. Proops DW, Donaldson I, Cooper HR, et al. Outcomes from adult implantation, the first 100 patients. J Laryngol Otol Suppl. 1999;24:5-13.

7. Blamey P, Arndt P, Bergeron F, et al. Factors affecting auditory performance of postlinguistically deaf adults using cochlear implants. Audiol Neurootol. 1996;1: 293-306.

8. O'Donoghue GM, Nikolopoulos TP, Archbold SM. Determinants of speech perception in children after cochlear implantation. Lancet. 2000;356:466-468. 
9. Miyamoto RT, Osberger MJ, Todd SL, et al. Variables affecting implant performance in children. Laryngoscope. 1994;104:1120-1124.

10. Sarant JZ, Blamey PJ, Dowell RC, Clark GM, Gibson WP. Variation in speech perception scores among children with cochlear implants. Ear Hear. 2001;22: $18-28$.

11. Kujala T, Alho K, Naatanen R. Cross-modal reorganization of human cortical functions. Trends Neurosci. 2000;23:115-120.

12. Veit P, Bizaguet G. Detection of auditory reactions in newborn infants [in French]. Rev Laryngol Otol Rhinol (Bord). 1968;89:433-439.

13. Giraud AL, Price CJ, Graham JM, Truy E, Frackowiak RS. Cross-modal plasticity underpins language recovery after cochlear implantation. Neuron. 2001;30:657-663.

14. Giraud AL, Truy E. The contribution of visual areas to speech comprehension: a PET study in cochlear implants patients and normal-hearing subjects. Neuropsychologia. 2002;40:1562-1569.

15. Giraud AL, Truy E, Frackowiak RS, Gregoire MC, Pujol JF, Collet L. Differential recruitment of the speech processing system in healthy subjects and rehabilitated cochlear implant patients. Brain. 2000;123:1391-1402.

16. Wong D, Miyamoto RT, Pisoni DB, Sehgal M, Hutchins GD. PET imaging of cochlear-implant and normal-hearing subjects listening to speech and nonspeech. Hear Res. 1999;132:34-42.

17. Naito Y, Okazawa H, Honjo I, et al. Cortical activation during sound stimulation in cochlear implant users demonstrated by positron emission tomography. Ann Otol Rhinol Laryngol Suppl. 1995;166:60-64.

18. Naito Y, Tateya I, Fujiki N, et al. Increased cortical activation during hearing of speech in cochlear implant users. Hear Res. 2000;143:139-146.

19. Green KM, Julyan PJ, Hastings DL, Ramsden RT. Auditory cortical activation and speech perception in cochlear implant users: effects of implant experience and duration of deafness. Hear Res. 2005;205:184-192.

20. Lee JS, Lee DS, Oh SH, et al. PET evidence of neuroplasticity in adult auditory cortex of postlingual deafness. J Nucl Med. 2003;44:1435-1439.

21. Lee HJ, Kang E, Oh SH, et al. Preoperative differences of cerebral metabolism relate to the outcome of cochlear implants in congenitally deaf children. Hear Res. 2005;203:2-9.

22. Mortensen MV, Madsen S, Gjedde A. Cortical responses to promontorial stimulation in postlingual deafness. Hear Res. 2005;209:32-41.

23. Belin P, Zatorre RJ, Lafaille P, Ahad P, Pike B. Voice-selective areas in human auditory cortex. Nature. 2000;403:309-312.

24. Belin P, Fecteau S, Bedard C. Thinking the voice: neural correlates of voice perception. Trends Cogn Sci. 2004;8:129-135.
25. Gervais $\mathrm{H}$, Belin $\mathrm{P}$, Boddaert $\mathrm{N}$, et al. Abnormal cortical voice processing in autism. Nat Neurosci. 2004;7:801-802.

26. Fox PT, Mintun MA, Raichle ME, Herscovitch P. A noninvasive approach to quantitative functional brain mapping with $\mathrm{H}_{2}{ }^{15} \mathrm{O}$ and positron emission tomography. J Cereb Blood Flow Metab. 1984;4:329-333.

27. Bendriem BCM, Dahlbom M, Trebossen R, et al. Evaluation of the ECAT EXACT HR+: a new positron camera with 2D/3D acquisition capabilities and nearly isotropic spatial resolution [abstract]. J Nucl Med. 1996;37(suppl):170P.

28. Talairach J, Tournoux P. Co-Planar Stereotaxic Atlas of the Human Brain: 3-Dimensional Proportional System-An Approach to Cerebral Imaging. New York, NY: Thieme Medical; 1988.

29. Friston KJ, Worsley KJ, Poline JB, Frith CD, Frackowiak RSJ. Statistical parametric maps in functional imaging: a general linear approach. Hum Brain Mapp. 1995;2:189-210.

30. Samson Y, Belin P, Thivard L, Boddaert N, Crozier S, Zilbovicius M. Auditory perception and language: functional imaging of speech sensitive auditory cortex [in French]. Rev Neurol (Paris). 2001;157:837-846.

31. Binder JR, Frost JA, Hammeke TA, et al. Human temporal lobe activation by speech and nonspeech sounds. Cereb Cortex. 2000;10:512-528.

32. Vouloumanos A, Kiehl KA, Werker JF, Liddle PF. Detection of sounds in the auditory stream: event-related fMRI evidence for differential activation to speech and nonspeech. J Cogn Neurosci. 2001;13:994-1005.

33. Zatorre RJ, Belin P. Spectral and temporal processing in human auditory cortex. Cereb Cortex. 2001;11:946-953.

34. Herzog H, Lamprecht A, Kuhn A, Roden W, Vosteen KH, Feinendegen LE. Cortical activation in profoundly deaf patients during cochlear implant stimulation demonstrated by $\mathrm{H}_{2}{ }^{15} \mathrm{O}$ PET. J Comput Assist Tomogr. 1991;15: 369-375.

35. Mortensen MV, Mirz F, Gjedde A. Restored speech comprehension linked to activity in left inferior prefrontal and right temporal cortices in postlingual deafness. Neuroimage. 2006;31:842-852.

36. Zatorre RJ, Belin P, Penhune VB. Structure and function of auditory cortex: music and speech. Trends Cogn Sci. 2002;6:37-46.

37. Scott SK, Blank CC, Rosen S, Wise RJ. Identification of a pathway for intelligible speech in the left temporal lobe. Brain. 2000;123:2400-2406.

38. Nadol JB Jr. Patterns of neural degeneration in the human cochlea and auditory nerve: implications for cochlear implantation. Otolaryngol Head Neck Surg. 1997; 117:220-228.

39. Mosnier I, Ambert-Dahan E, Smadja M, et al. Ann Otolaryngol Chir Cervicofac. 2006;123:71-78 